\title{
Effect of Stress Management in Over-weight and Obese Women
}

\author{
Dr. Shweta Kotwani *, Dr. Amita Mehta ${ }^{2}$ \\ P.T. School and Centre Seth G.S.Medical College and KEM Hospital, Parel, Mumbai \\ Address of correspondence - shweta.kotwani31528@gmail.com
}

\section{Introduction}

Obesity, with its co-morbidities, is a major public health problem. Excess body weight appears to arise from a dynamic interaction between an organism's biology, behavior and the increasingly obesogenic environment (Gasevic\&Vukm- irovich, 2011)

Unhealthy eating habits combined with a sedentary lifestyle, leading to a chronic surplus in energy, appear to comprise the key risk factors in the etiology of obesity Chronic exposure to stress may also play a role in the development of obesity, possibly through the hyper activation of the hypothalamic pituitary adrenocortical axis (HPA axis) and through appetite regulation (Vicennati\&Pasqui, 2009)

Various types of psychosocial stressors, such as occupational stress, have been significantly related to increased body mass index (BMI) and waist circumference. Similarly, other studies have shown that trait disorders, such as anxiety and depressive emotions , are also related to obesity. There is good evidence that stress eating occurs particularly in restrained eaters or those participants who diet, although not in those who are restricting their food intake. Restrained eating or dietary restraint is defined as an attempted weight regulation strategy, characterized by the exertion of cognitive control over food intake. Dietary restraint is a behavior that is assumed to be a psychological determinant of overeating and eating disorders, whereas dieting is the actual planned food restriction as part of an attempt to change eating behavior and to lose weight.

\section{Psychological aspects of obesity}

There has been considerable effort to find personality variables associated with obesity, however there is no evidence that obese people differ psychologically from non-obese people. There is no difference between obese and non-obese people in the following characteristics: degree of depression (Stewart 1983), the incidence of psychopathology (Friedman 1995; Stunkard 1992), social adjustment (Sallade 1973), 'traits' of masculinity-femininity, locus of control, assertiveness and self-consciousness (Klesges 1984) and personality type (Blackmeyer 1990). However, obese people in general do not find their state desirable. For example, in a sample of formerly obese people who had undergone gastric surgery, Rand and MacGregor (Rand 1991) found that all of the 47 participants who were interviewed would rather be deaf, dyslexic, diabetic, or suffer bad heart disease or acne than return to being morbidly overweight. Forty-two percent preferred blindness to obesity, and 43 participants would rather have a leg amputated. This dislike of obesity felt by sufferers may reflect stigmatization by others in the population. Diverse groups hold negative stereotypes of obese people. Boys between six and ten years old rate silhouettes of obese boys as someone who would fight, cheat, get teased and lie, and who was lazy, sloppy, naughty, mean, ugly, dirty and stupid (Staffieri 1967). Studies of adult attitudes similarly demonstrate negative attitudes. Adult hospital outpatients rate silhouettes of an overweight child as less likeable than a child with a deformed leg, with a missing hand, with a facial deformity, or who was confined to a wheelchair (Maddox 1968). Similarly, doctors and medical students hold negative views of people who are obese. Medical students rate overweight women as less likeable, more emotional, and less likely to benefit from treatment. Doctors rate overweight patients as weak-willed, ugly and awkward (Bretyspraak 1977; Maddox 1969). Job prospects are also affected. Larkin and Pines (Larkin 1979) showed that overweight candidates were less likely to be hired, even though equally competent on job-related tests. Given these negative attitudes, it is surprising that obese people are not more likely to be depressed or to have psychopathology. Because studies comparing obese and non-obese persons have generally failed to find differences in global aspects of psychological functioning, the resulting conclusion has been that obesity is not a risk factor for psychological problems. This is at odds with clinical impression, reports from overweight people, and a consistent literature showing strong cultural bias and negative attitudes toward obese people (Friedman 1995). It is clear that obesity confers negative consequences on both the physical and psychosocial aspects of quality of life, especially among the severely obese. Therefore, the lack of evidence supporting the existence of psychological morbidity in obese people is likely to be a reflection of the limitations of studies performed to date rather than an accurate reflection of the psychological well-being of individuals who are obese. Also, studies which demonstrate that the effects of weight loss appear to be psychologically favorable with improved self-esteem, social functioning and sense of wellness support the notion that excess weight is associated with higher levels of psychological morbidity than normal weight (Kushner 2000).

\section{Subjects and Methods}




\section{International Journal of Innovative Research in Medical Science (IJIRMS) Volume 04 Issue 01 Jan 2019, ISSN: 2455-8737, Imp. Factor - 4.102 \\ Available online at $-\underline{w w w . i j i r m s . i n}$}

\section{Inclusion of subjects}

- Overweight and obese women BMI 25-29 kg/m2 and > or equal to $30 \mathrm{~kg} / \mathrm{m} 2$ respectively.

- Age group of 25-55 years.

- Individuals willing to participate in the study.

- Participants were followed up for at least 4 weeks.

\section{Exclusion of subjects}

- Previously diagnosed with mental or psychiatric disorders or conditions, such as depression or eating disorders.

- Underweight women or women with normal BMI that is less than $25 \mathrm{~kg} / \mathrm{m} 2$.

\section{Procedure}

The present study comparing a 4-week self-administered stress management programme, applied concurrently with a weight-loss dietary regime, to a group of obese women. Changes in eating behavior, body weight and perceived stress in women were measured before and after the intervention.

All the participants visited the clinic intending to lose weight. Participants who met the inclusion criteria were provided with detailed information about the study and provided their written consent to participate. All the participants were placed on a balanced diet and encouraged to consume low fat dairy, more fruits and vegetables, whole grains and reduce sugars and saturated fat. The weight-loss clinic was based at the outpatient's department (OPD) of a large public hospital in Parel, Mumbai, where, as a result of limited resources, the prescribed weight-loss diet offered is generic. It was based on promoting good eating habits, motivation for lifestyle changes and the suggestion of a low-energy eating plan and also a proper weight loss regime which included aerobic exercise session which was planned by the clinical physiotherapist. The participants attended a 40-min individual session of the stress management intervention. The stress management session included learning techniques for progressive muscular relaxation and diaphragmatic breathing (benson, 1982). The participants were informed about the technique and given a audio clip with recorded instructions and were instructed to practice it twice a day for 4 weeks.

Baseline assessments included anthropometric data. Body weight and height were recorded for each participant prior to start of the study and again at the end. Body weight was recorded with the use of a digital scale and with participants standing without shoes. Height was also measured with participants standing without shoes, keeping their shoulders in a relaxed position with the use of a measuring tape. BMI was calculated by dividing weight $(\mathrm{kg})$ with standing height squared $(\mathrm{m})$ and the classification was according to the current who definitions for overweight and obesity as BMI 25$29 \mathrm{~kg} / \mathrm{m} 2$ and > or equal to $30 \mathrm{~kg} / \mathrm{m} 2$, respectively (who, 2000).

\section{Waist/Hip Ratio (WHR)}

The pattern of body weight distribution is recognized as an important predictor of health risks of obesity. Thus, the measurement of waist circumference is used as another indicator of obesity. This measurement is used to identify those with the abdominal type of obesity associated with greater health risk. The waist circumference is typically measured at the smallest circumference above the umbilicus and below the xiphoid process.
The technician should stand on the right side of the client. The measurement should be made on bare skin. The measurement should be taken at the end of a normal exhalation by the client.

The measuring tape should be parallel to the floor and should be pulled to lay flat on the skin without compressing the skin (some measurement tapes have a gauge to standardize the tension). Multiple measurements should be taken to determine the smallest circumference site. The mean of two measurements at this location (that do not differ by more than $1 \mathrm{~cm}$ ) is used. Some have advocated the additional measurement of the hip circumference to assess body fat distribution. The two circumference values are used to determine the waist-to hip ratio (WHR). The hip circumference is measured as the largest circumference around the buttocks, above the gluteal fold (posterior extension), following the same standardization procedures used for waist measurement.

Waist circumference $>$ or equal to $88 \mathrm{~cm}$ and WHR $>0.85$ (American College of Sports Medicine. ACSM's Guidelines for Exercise Testing and Prescription, 8th ed. Philadelphia (PA): Wolters Kluwer Health Ltd: 2009, 63 p.)

\section{Eating Attitudes Test (EAT-26)}

The EAT-26 (Garner \&Garfinkel, 1979) is a widely used selfreport measure for assessment of disordered eating attitudes and behavior. It contains three subscales: dieting, bulimia and food preoccupation and oral control. The scores are ranked on a sixpoint scale from always to never, with three points allotted to 'always', two points to 'very often', one point to 'often', and 0 points to the others. Total EAT-26 scores are derived as a sum of the composite items, and range from 0 to 78. EAT-26 scores >or equal to 20 are frequently associated with abnormal eating attitudes and behavior, and may identify individuals with an eating disorder. The questionnaire had been previously validated (Stalikas\&Roussi, 2008).

\section{Perceived Stress Scale (PSS-14)}

The PSS consists of 14 items (PSS-14), seven positive and seven negative. The negative element is intended to assess lack of control and negative affective reactions, whereas the positive element measures the degree of ability to cope with existing stressors. Each item is rated on a five-point scale from ' $0=$ never' to ' $4=$ very often', covering the preceding month. Total scores are calculated after reversing positive items scores and then summing up all scores. A higher score indicates greater stress. The questionnaire had been previously validated in the Greek language (Andreou \& Alexopoulos, 2011)

\section{Statistics}

Data was tabulated, examined and analyzed using Microsoft Excel Software 2010. Comparison between pre and post intervention based on demographic and baseline variables were examined using Students t-test.

\section{Results}

Table 1: Table shows the results of baseline comparisons between pre and post interventional scores. Data collection of 14 overweight and obese women was obtained. Analysis was done using comparative t-test and $\mathrm{p}$ value was obtained. $\left({ }^{*} \mathrm{p}<0.05\right.$, S.D standard deviation, BMI body mass index, W/H Ratio waist hip ratio, PSS-14 perceived stress scale, EAT-26 eating attitude test). 
International Journal of Innovative Research in Medical Science (IJIRMS)

Volume 04 Issue 01 Jan 2019, ISSN: 2455-8737, Imp. Factor - 4.102

Available online at - $\underline{w w w . i j i r m s . i n}$

\begin{tabular}{|lcccc|} 
& Mean & S.D & T-test & P-value \\
\hline Weight & 67.8 & 9.09 & 1.77093 & $0.000021886^{*}$ \\
\hline BMI & 29.1 & 3.34 & 1.7709 & $0.002454^{*}$ \\
\hline $\begin{array}{l}\text { Waist } \\
\text { Circumference }\end{array}$ & 92.9 & 9.01 & 1.7709 & $0.000516^{*}$ \\
\hline W/H Ratio & 0.84 & 0.07 & 1.7709 & $0.067624^{\mathrm{NS}}$ \\
\hline PSS-14 & 25.93 & 4.74 & 1.7709 & $0.000328^{*}$ \\
\hline EAT-26 & 14.32 & 3.70 & 1.7709 & $0.16778063^{\mathrm{NS}}$ \\
\hline
\end{tabular}

*S: Significant NS: Not Significant

The study included $100 \%$ women out of which $57 \%$ were overweight and $43 \%$ were obese. Table 1 shows baseline data for the 14 participants who completed the Stress Management programme. The results indicated significant differences in weight loss post the intervention. Participants lost a mean of $0.43571 \mathrm{~kg}$ $(\mathrm{p}<0.05)$ during the 4 weeks of the intervention. Significant differences were also found in BMI of $0.265 \mathrm{~kg} / \mathrm{m} 2(\mathrm{p}<0.05)$. The post intervention group scored lower in the PSS-14 after 4 weeks of intervention by $1.8571 \quad(\mathrm{p}<0.05)$. The results indicated differences in the eating behavior by 0.07143 in EAT-26, although there was no statistical evidence to suggest any difference as $\mathrm{p}=$ 0.16778. Regarding the remaining variables, there were no differences detected.

\section{Discussion}

The possible role of stress management on the efficacy of weightloss programmes has not been adequately investigated previously because only a few studies have used stress management interventions combined with dietary treatment (Manzoni \& Pagnini, 2009). The present study was designed to evaluate the efficacy of a 4-week stress management programme on weight loss and eating behavior in a sample of overweight and obese women who start a weight-loss programme. The findings of the present study demonstrate a significant improvement in weight loss and perceived stress levels in the SM group after the intervention. However, there was no significant difference on eating behavior post intervention.

It is important to clarify that dieting and dietary restraint are not equivalent. There is evidence that dietary restraint may contribute to successful weight management more than dieting (Rideout \& Barr, 2009). In a recent study examining the complex interactive impact of major stress and minor stressors on the relation between dietary restraint and binge eating, hierarchal linear regression revealed a significant three-way interaction between dietary restraint, life event stress and daily stress (Woods \& Racine, 2010).

In addition, successful weight loss has been associated with increased dietary restraint in obese patients seeking weight-loss treatment (Dalle Grave \& Calugi, 2009). According to Savage \& Hoffman (2009), dietary restraint is defined as a conscious control of food intake and is related to lower body mass indices. On the other hand, dieting is rarely clearly defined and is associated with weight gain through triggering overeating (Gorin \& Phelan, 2004). It is well documented that greater dietary restraint is associated with increased urinary cortical levels, a biological marker of stress (McLean \& Barr, 2001).

Stress and negative emotions have also been shown to be critical factors in inducing overeating as a form of maladaptive coping in

some patients with obesity (Manzoni \& Pagnini, 2009). Previous studies suggest that emotional eating may be a crucial target for intervention on stress-related food consumption, particularly regarding the frequency of snacking and choice of foods (Wallis \& Hetherington, 2009). There is evidence that the HPA axis may influence eating behavior through pathways that prominently control dietary intake among obese individuals. For example, HPA axis activation modifies the incentive salience of food rewards (Adam \& Epel, 2007), which is assumed to be a strong influence on energy intake among obese individuals (Mela, 2006). Moreover, appetite is regulated by stress (Torres \& Nowson, 2007; Wardle, 2007) and physiological mechanisms, including hormonelike peptides or hormones. Such peptides are neuropeptide- Y and ghrelin (Chaudhri \& Small, 2006) which stimulate appetite. On the other hand, leptin is an important hormone that can reduce food intake by suppressing appetite (Blundell \& Goodson, 2001). The PSS was selected for assessment of the stress levels of the individuals. It is interesting to note that the PSS detected significant differences in stress levels.

\section{Conclusion}

In conclusion, this study was done to demonstrate that a stress management intervention in combination with a conventional dietary intervention can contribute to greater weight loss in overweight and obese women who follow a weight-loss dietary treatment programme.

\section{References}

[1] Adam, T.C. \& Epel, E.S. (2007) Stress, eating and the reward system. Physiol. Behav. 91.

[2] Anderson, J.W., Konz, E.C., Frederich, R.C. \& Wood, C.L.(2001) Long-term weight-loss maintenance: a metaanalysisof US studies. Am. J. Clin. Nutr. 74.

[3] Andreou, E., Alexopoulos, E.C., Lionis, C., Varvogli, L, Gnardellis, C., Chrousos, G.P. \& Darviri, C. (2011) Perceived stress scale: reliability and validity study in Greece. Int. J. Environ. Res. Public Health 8.

[4] Benson, H. (1982) The relaxation response: history, physiological basis and clinical usefulness. Acta. Med. Scand. Suppl.

[5] Bjorvell, H., Aly, A., Langius, A. \& Nordstr€om, G. (1994) Indicators of changes in weight and eating behaviour in severely obese patients treated in a nursing behavioural program. Int. J. Obes. Relat. Metab. Disord. 18.

[6] Blundell, J.E., Goodson, S. \& Halford, J.C. (2001) Regulation of appetite: role of leptin in signalling systems for drive and satiety. Int. J. Obes. Relat. Metab. Disord. 25(Suppl.

[7] Bouras, G. \& Lykouras, L. (2011) The economic crisis and its impact on mental health. Encephalos.

[8] Bradshaw, A.J., Horwath, C.C., Katzer, L. \& Gray, A. (2009) Non-dieting group interventions for overweight and obese women: what predicts non-completion and does completion improve outcomes? Public Health Nutr. 13.

[9] Brunner, E.J., Chandola, T. \& Marmot, M.G. (2007) Prospective effect of job strain on general and central obesity in the Whitehall II Study. Am. J. Epidemiol. 165. 
[10] Chaudhri, O., Small, C. \& Bloom, S. (2006) Gastrointestinal hormones regulating appetite. Philos. Trans. R. Soc. Lond. B Biol. Sci. 367.

[11] Costarelli, V., Antonopoulou, K. \& Mavrovounioti, Ch. (2011) Psychosocial characteristics in relation to disordered eating attitudes in greek adolescents. Eur. Eat. Disord. Rev. 19.

[12] Costarelli, V., Demerzi, M. \& Stamou, D. (2009) Disordered eating attitudes in relation to body image and emotional intelligence in young women. J. Hum. Nutr. Diet. 22.

[13] Dalle Grave, R., Calugi, S., Corica, F., Di Domizio, S. \& Marchesini, G. (2009) Psychological variables associated with weight loss in obese patients seeking treatment at medical centers. J. Am. Diet. Assoc. 109.

[14] Freeman, L.M. \& Gil, K.M. (2004) Daily stress, coping, and dietary restraint in binge eating. Int. J. Eat. Disord. 36.

[15] Garner, D.M. \& Garfinkel, P.E. (1979) The eating attitudes test: an index of the symptoms of anorexia nervosa. Psychol. Med. 9.

[16] Garner, D.M., Olmsted, M.P., Bohr, Y. \& Garfinkel, P.E. (1982) The eating attitudes test: psychometric features and clinical correlates. Psychol. Med. 12.

[17] Gasevic, D., Vukmirovich, I., Yusuf, S., Teo, K., Chow, C., Dagenais, G. \& Lear, S.A. (2011) A direct assessment of 'obesogenic' built environments: challenges and recommendations. J. Environ. Public Health 2011, 161574.

[18] Gorin, A.A., Phelan, S., Wing, R.R. \& Hill, J.O. (2004) Promoting long-term weight control: does dieting consistency matter? Int. J. Obes. Relat. Metab. Disord. 28.

[19] Grotz, M., Hapke, U., Lampert, T. \& Baumeister, H. (2011) Health locus of control and health behaviour: results from a nationally representative survey. Psychol. Health Med. 16.

[20] Jeffery, R.W., Drewnowski, A., Epstein, L.H., Stunkard, A.J., Wilson, G.T., Wing, R.R. \& Hill, D.R. (2000) Long-term maintenance of weight loss: current status. Health Psychol. 19(Suppl. 1).

[21] Katzer, L., Bradshaw, A.J., Horwath, C.C., Gray, A.R., O'Brien, S. \& Joyce, J. (2008) Evaluation of a 'nondieting' stress reduction program for overweight women: a randomized trial. Am. J. Health Promot. 22.

[22] Kuiper, N.A., Olinger, L.J. \& Lyons, L.M. (1986) Global perceived stress level as a moderator of the relationship between negative life events and depression. J. Human Stress 12.

[23] Lee, E.S., Kim, Y.H., Beck, S.H., Lee, S. \& Oh, S.W. (2005) Depressive mood and abdominal fat distribution in overweight premenopausal women. Obes. Res. 13.

[24] Manzoni, G.M., Pagnini, F., Gorini, A., Preziosa, A., Castelnuovo, G., Molinari, E. \& Riva, G. (2009) Can relaxation training reduce emotional eating in women with obesity? An exploratory study with 3 months of follow-up. J. Am. Diet. Assoc. 109.

[25] Manzoni, G.M., Gorini, A., Preziosa, A., Pagnini, F., Castelnuovo, G., Molinari, E. \& Riva, G. (2008) New technologies and relaxation: an explorative study on obese patients with emotional eating. J. Cyber. Ther. Rehabil. 1. 